\title{
Correlación del índice de severidad por tomografía y pronóstico de pacientes con neumonía por COVID-19
}

\author{
Correlation of the severity index by tomography and \\ prognosis of patients with COVID-19 pneumonia
}

\author{
Estefanía Murrieta-Peralta, * Ana Patricia Chischistz-Condey, \\ Karina Iliana Holguin-Andrade,* Arturo Cadena-Fernández,* Hugo Alberto Cervantes-Flores, ${ }^{*}$ \\ Jorge Ramírez-Landero, Héctor Murrieta-González*
}

*Centro Médico ABC, Ciudad de México.

RESUMEN. Introducción: La neumonía por COVID-19 es una enfermedad infecciosa causada por un síndrome respiratorio agudo secundario a coronavirus 2. Se ha propuesto que los hallazgos tomográficos de pacientes con sospecha de infección por COVID-19 sean subclasificados. Objetivo: Medir la correlación entre el índice de severidad por tomografía de tórax, así como el grado de afección pulmonar por tomografía y el pronóstico de los pacientes con infección por COVID-19. Material y métodos: Se trata de un estudio observacional analítico retrospectivo en el que se analizó a un total de 233 pacientes. Conclusión: El índice de severidad podría ser empleado para predecir pacientes que requerirán ventilación mecánica. El porcentaje de volumen pulmonar afectado y respetado fue útil para predecir el ingreso a la UTI, el uso de ventilación mecánica, los días de estancia intrahospitalaria y el desenlace.

Palabras clave: COVID-19, índice de severidad por tomografía, volumen pulmonar afectado, volumen pulmonar respetado, pronóstico.

\section{INTRODUCCIÓN}

La neumonía por COVID-19 es una enfermedad altamente infecciosa causada por un síndrome respiratorio agudo secundario a coronavirus 2 (SARS-CoV-2). Esta enfermedad fue reportada por primera vez en la provincia de Wuhan, China, en diciembre de 2019, y desde ese momento se ha propagado a otras ciudades y países.

\section{Correspondencia:}

\section{Dra. Estefanía Murrieta Peralta}

Centro Médico ABC, Ciudad de México.

Correo electrónico: emurrietap@gmail.com

Recibido: 03-I-2021; Aceptado: 20-I-2021.

Citar como: Murrieta-Peralta E, Chischistz-Condey AP, Holguin-Andrade KI, Cadena-Fernández A, Cervantes-Flores HA, Ramírez-Landero J, et al. Correlación del índice de severidad por tomografía y pronóstico de pacientes con neumonía por COVID-19. Neumol Cir Torax. 2021; 80 (1): 19-28. https:// dx.doi.org/10.35366/99450
ABSTRACT. Introduction: COVID-19 pneumonia is a highly infectious disease caused by a respiratory syndrome secondary to coronavirus 2 . It has been proposed that the tomographic findings of these patients with suspected COVID-19 infection be subclassified. Objective: To measure the correlation between the chest tomography severity index as well as the degree of pulmonary involvement by tomography and the prognosis of patients with COVID-19 infection. Material and methods: This is a retrospective analytical observational study in which a total of 233 patients were analyzed. Conclusion: We conclude that the severity index could be used to predict patients who will require mechanical ventilation. The percentage of lung volume affected and respected was useful to predict admission to the ICU, use of mechanical ventilation, days of hospital stay and the outcome.

Keywords: COVID-19, severity index, affected lung volume, respected lung volume, prognosis.

En enero de 2020 fue declarada como una emergencia mundial en el campo de la salud pública por la Organización Mundial de la Salud (OMS). ${ }^{1}$ Al momento en que escribimos este manuscrito se ha reportado la incidencia de esta enfermedad en 191 países y más de 67 millones de casos mundialmente..$^{2-4}$

Hasta ahora, el estándar para el diagnóstico es la prueba de reacción en cadena de la polimerasa transcriptasa reversa en tiempo real (RT-PCR, por sus siglas en inglés) de los ácidos nucleicos virales. ${ }^{5}$ Sin embargo, se han obtenido distintos estudios en los que la tomografía computarizada (TC) cobra importancia para el diagnóstico de pacientes con prueba RT-PCR negativa, reportando sensibilidades de hasta $98 \%$ para la tomografía. ${ }^{6}$

Los hallazgos tomográficos descritos comúnmente son zonas de vidrio esmerilado con tendencia a la consolidación, distribución periférica y de predominio en lóbulo medio y lóbulo inferior derecho. ${ }^{7}$ Con base en la experiencia acumulada en los últimos meses, se ha dado la recomendación de 
que casos con sospecha clínica alta, historia de exposición y hallazgos típicos en la tomografía computarizada pueden tratarse como neumonía por COVID-19. ${ }^{8}$

En relación con esto la Sociedad Fleischner publicó un consenso multinacional en abril de 2020, en el que definen diferentes escenarios frente a los cuales la tomografía computarizada puede tener una diferente rentabilidad y en la que se declara que realizar radiografías de tórax como diagnóstico y seguimiento de los casos es insuficiente e incrementa el riesgo de exposición a rayos X. Estos escenarios se definen de acuerdo con los hallazgos clínicos (enfermedad leve, moderada o grave), la probabilidad preprueba que dependerá del riesgo de exposición de cada individuo y la disponibilidad de recursos para la realización de la prueba RT-PCR. ${ }^{9}$

Se ha propuesto que los hallazgos tomográficos de dichos pacientes con sospecha de infección por COVID-19 sean subclasificados. Uno de los más empleados a nivel mundial y que actualmente utilizamos en el Centro Médico $\mathrm{ABC}$ son las cuatro categorías propuestas por la Sociedad Norteamericana de Radiología, Colegio Americano de Radiología y Sociedad Torácica de Radiología (en patrón típico, indeterminado, atípico y negativo)..$^{10}$ Éste sólo funciona para clasificar a los pacientes y no para conferir un riesgo de progresión.

Se han descrito varias formas para poder llevar a cabo el seguimiento de los pacientes con infección por COVID-19 y medir de forma objetiva la progresión acorde a los signos tomográficos. Se han propuesto métodos cuantitativos y semicuantitativos para conferir un puntaje de severidad a pacientes con neumonía por COVID-19, que ya habían sido empleados para síndrome de dificultad respiratoria ocasionado por otras etiologías como virus de influenza. ${ }^{11}$ Entre ellos uno de los más importantes es el índice de severidad por tomografía de tórax (CT-SS, por sus siglas en inglés), en el que se asigna una puntuación acorde a la afección de cada segmento pulmonar (cero sin afección, uno menos de $50 \%$ y dos más de $50 \%$ de afección del segmento) y con lo que obtenemos un puntaje teórico entre cero y 40 (de acuerdo con la suma de afección de cada uno de los 20 segmentos en los que se dividen ambos pulmones). Dicho índice ha demostrado tener una sensibilidad de hasta $83.3 \%$ y especificidad de $94 \% .^{12}$

Existen otros métodos para medir la severidad del cuadro acorde a los hallazgos tomográficos. Por ejemplo, uno que es muy parecido al descrito previamente es el puntaje de severidad total (TSS, por sus siglas en inglés), con una sensibilidad de $82.6 \%$ y especificidad de $100 \%{ }^{13}$ En éste los hallazgos encontrados se dividen por lóbulos y no por segmentos pulmonares. Cada uno de los lóbulos pulmonares se evalúa con un puntaje de afección: $0(0 \%)$, 1 (1-25\%), 2 (26-50\%), 3 (51-75\%) o 4 (76-100\%), con lo que se obtiene un puntaje teórico entre cero y 20 (acorde a la suma de afección mínima y máxima por cada uno de los cinco lóbulos pulmonares). Este puntaje suele ser más difícil de aplicar debido a su alta variabilidad interobservador.

Hoy en día, contamos con herramientas cuantitativas como la propuesta por Colombi et al. ${ }^{14}$ en el que determinan, mediante una cuantificación visual combinada a una cuantificación por software, el volumen pulmonar afectado y el volumen adecuadamente neumatizado. Con la realización de esta cuantificación en pacientes confirmados con infección por COVID-19 demostraron que existe una correlación entre el volumen pulmonar mal neumatizado y el riesgo de ingreso a la Unidad de Terapia Intensiva (UTI) e incremento en la mortalidad. La cuantificación visual que utilizaron se trata de la descrita por Ichikado et al., que está aprobada para su utilización en pacientes con síndrome de dificultad respiratoria, pero que requiere de un radiólogo experto en interpretación de tórax para poder evaluarla..$^{15}$ Más aún, a pesar de que el software empleado es una herramienta gratuita, se trata de un método que requiere de alguien experto en el manejo del mismo y aún no ha sido aprobado para su uso clínico.

Todos los sistemas de los que hemos hablado hasta este momento emplean como medidas de pronóstico el ingreso a la UTI y la mortalidad para evaluar su rendimiento. Existen calculadoras que estratifican el riesgo para ingreso a la UTI como la publicada por la Sociedad Americana de Enfermedades Infecciosas (IDSA) y la Sociedad Torácica Americana (ATS) para el manejo de neumonías adquiridas en la comunidad. El fin de ésta es identificar a los pacientes que requieren ingreso hospitalario o manejo ambulatorio. Con los criterios propuestos, si el paciente cumple con un criterio mayor o tres menores, se trata de una neumonía severa que requiere de manejo intrahospitalario en la UTI. ${ }^{16}$ Otra herramienta es el puntaje MuLBSTA utilizado como predictor para evaluar el riesgo de mortalidad a 90 días en una neumonía viral. Esta herramienta clínica puede estratificar con precisión a pacientes hospitalizados para la toma de decisiones clínicas adicionales al manejo. ${ }^{17}$ Sin embargo, este puntaje aún no se encuentra validado para neumonía por COVID-19. Otro instrumento empleado como el MuLBSTA para calcular mortalidad es el índice de severidad de neumonía (PSI, por sus siglas en inglés), que también se conoce como la puntuación PORT. Esta escala se obtuvo con base en datos de 14,199 pacientes adultos y posteriormente se validó en más de 40,000 pacientes en Estados Unidos y Canadá. ${ }^{18}$ Al comparar con el puntaje MuLBSTA, PSI es empleada en pacientes con neumonía para estimar la mortalidad y categorizarlos en cinco grupos de riesgo. En la actualidad, estas tres escalas (IDSA/ATS, MuLBSTA y PSI) son empleadas en el paciente con neumonía por COVID-19, aunque ninguna está validada oficialmente.

Además, existen otras variables importantes que definen el pronóstico a corto plazo de los pacientes como son la 
saturación de oxígeno $\left(\mathrm{SaO}_{2}\right)$ y la presencia de reactantes de fase aguda (interleucina 6 [IL-6], proteína $C$ reactiva $[P C R]$, dímero $D[D D]$, procalcitonina $[P C T]$, ferritina, entre otras) relacionadas a la severidad del cuadro clínico e indicación de intubación como método de ventilación mecánica invasiva. ${ }^{19}$

Al día de hoy, en el Centro Médico $\mathrm{ABC}(\mathrm{CMABC})$ hemos atendido a pacientes con neumonía por COVID-19 y han sido manejados de acuerdo con los estándares mundiales de calidad y servicio hospitalario. De este modo, reconocemos que en el Servicio de Imagenología de este hospital hemos estado en actualización continua y revisando las recomendaciones de las sociedades radiológicas más reconocidas a nivel mundial. Por lo que hemos implementado el reporte estructurado acorde a cuatro categorías para informar hallazgos acerca de pacientes con sospecha de infección por COVID-19. En este trabajo queremos ir más allá y evaluar si el uso de una herramienta sencilla de estratificación de riesgo por tomografía computarizada es realmente aplicable en nuestra población y si tiene un valor pronóstico en cuanto a ingreso a UTI, progresión a ventilación mecánica invasiva y mortalidad.

\section{MATERIAL Y MÉTODOS}

\section{Objetivo}

Medir la correlación entre el índice de severidad por tomografía de tórax (CT-SS), así como el grado de afección pulmonar por tomografía y el pronóstico de los pacientes con infección por COVID-19.

\section{Objetivos específicos}

1. Medir la correlación entre el CT-SS, así como el grado de afección pulmonar por tomografía y el ingreso de pacientes con infección por COVID-19 a la UTI, el uso de ventilación mecánica invasiva, días de estancia intrahospitalaria y mortalidad.

\section{Población}

Pacientes mayores de 18 años con cuadro confirmado por RT-PCR o sospechoso (datos clínicos y hallazgos característicos en TC) que cuentan con tomografía computarizada de tórax inicial entre diciembre de 2019 y agosto de 2020.

Se excluyeron pacientes con tomografías computarizadas de tórax iniciales no valorables por presencia de artefacto de movimiento o artefactos metálicos externos, pacientes con pérdida de seguimiento para poder evaluar las variables pronósticas y pacientes que no desearon realizarse laboratorios en $\mathrm{CMABC}$ o que tienen laboratorios externos.

\section{Método}

Se trata de un estudio observacional analítico retrospectivo realizado en el CMABC, en el que se evaluarán las tomografías computarizadas de tórax de ingreso de los pacientes con infección por COVID-19. Las tomografías serán evaluadas por dos médicos radiólogos adscritos expertos, quienes darán un índice de severidad por tomografía computarizada, que se correlacionará con variables pronósticas. También se realizará una medición del grado de volumen pulmonar afectado mediante un software que se correlacionará con las mismas variables pronósticas.

\section{Análisis estadístico}

El análisis estadístico se llevó a cabo mediante el software SPSS v25 (IBM). Las variables continuas con distribución normal se presentan con medias y desviación estándar. Las variables continuas (Tabla 1) con distribución no estándar se presentan en medianas y rango intercuartílico (RIC). Para las variables cualitativas (Tabla 1) se presenta su frecuencia en porcentaje. Se realizarán las pruebas de Wilcoxon y $\chi^{2}$, así como correlaciones de Pearson y Spearman dependiendo de la distribución de las variables involucradas. Se considerará una p menor a 0.05 como estadísticamente significativa.

\section{RESULTADOS}

Las características de la población se muestran en la tabla 2.

El índice de severidad calculado por tomografía, mostró una media de $20.2 \pm 10.8$. La mediana de estancia hospitalaria fue de nueve días ( $\mathrm{RIC}=5-17)$. De los pacientes que requirieron intubación, la mediana fue de nueve días $(\mathrm{RIC}=7-14)$.

Respecto a la escala clínica de acuerdo al MuLBSTA, la mediana fue de siete $(\mathrm{RIC}=7-9)$, mientras que la mortalidad a 90 días por este método obtuvo una mediana de 3.9 $(\mathrm{RIC}=2.17-7.03)$. De acuerdo al puntaje de PSI, se obtuvo una mediana de 57 ( $\mathrm{RIC}=43.2-77.7)$.

Para las comorbilidades, un total de 104 pacientes $(44.6 \%)$ refirieron no padecer ninguna enfermedad, 36 (15.5\%) refirieron tener dos o más comorbilidades, 34 (14.6\%) padecían de hipertensión arterial sistémica, 19 (8.2\%) padecían de obesidad y 17 (7.3\%) padecían diabetes mellitus. Las medidas de tendencia central y dispersión de los valores por laboratorio (Tabla 3) y valores obtenidos por volumetría a partir de las tomografías (Tabla 4) se encuentran descritos a continuación.

\section{Grado de afección del parénquima pulmonar}

El grado de afectación pulmonar se midió al realizar volumetrías semiautomáticas de las tomografías computarizadas de 
Tabla 1: Variables de estudio.

\begin{tabular}{|c|c|c|c|}
\hline Variable & Tipo de variable & Escala de medición & Unidad de medición \\
\hline Edad & Cuantitativa discreta & Escala de razón & Unidad en años \\
\hline Sexo & Cualitativa nominal & Escala nominal & $\begin{array}{l}\text { 0: Hombre } \\
\text { 1: Mujer }\end{array}$ \\
\hline Comorbilidad & Cualitativa nominal & Escala nominal & $\begin{array}{l}\text { 0: Ninguna } \\
\text { 1: Hipertensión arterial } \\
\text { 2: Obesidad } \\
\text { 3: Diabetes mellitus } \\
\text { 4: Enfermedad pulmonar obstructiva crónica o asma } \\
\text { 5: Cáncer } \\
6: \text { Suma dos o más }\end{array}$ \\
\hline $\begin{array}{l}\text { Motivo por el que es referido } \\
\text { para realizar tomografía }\end{array}$ & Cualitativa nominal & Escala nominal & $\begin{array}{l}\text { 1: Sospecha de COVID-19 en triaje respiratorio } \\
\text { 2: Síntomas respiratorios y paciente ambulatorio } \\
\text { 3: Paciente en protocolo prequirúrgico }\end{array}$ \\
\hline RT-PCR & Cualitativa nominal & Escala nominal & $\begin{array}{l}\text { 0: No tiene } \\
\text { 1: Positiva } \\
\text { 2: Negativa }\end{array}$ \\
\hline $\begin{array}{l}\text { Reporte de hallazgos en } \\
\text { radiografía de tórax inicial }\end{array}$ & Cualitativa nominal & Escala nominal & $\begin{array}{l}\text { 1: Típico } \\
\text { 2: Indeterminado } \\
\text { 3: Atípico } \\
\text { 4: Negativo }\end{array}$ \\
\hline $\begin{array}{l}\text { Hallazgos de radiografía } \\
\text { de tórax inicial }\end{array}$ & Cualitativa nominal & Escala nominal & $\begin{array}{l}\text { 1: Normal } \\
\text { 2: Atelectasia, hiperinsuflación o bronquiectasias } \\
\text { 3: Consolidación un solo lóbulo } \\
\text { 4: Consolidación multifocal dos lóbulos } \\
\text { 5: Consolidación difusa en ambos pulmones }\end{array}$ \\
\hline $\begin{array}{l}\text { Reporte de hallazgos en } \\
\text { tomografía computarizada de } \\
\text { tórax inicial }\end{array}$ & Cualitativa nominal & Escala nominal & $\begin{array}{l}\text { 1: Típico } \\
\text { 2: Indeterminado } \\
\text { 3: Atípico } \\
\text { 4: Negativo }\end{array}$ \\
\hline $\begin{array}{l}\text { Índice de severidad por } \\
\text { tomografía de tórax }\end{array}$ & Cuantitativa discreta & Escala de razón & Numérico \\
\hline $\begin{array}{l}\text { Lóbulos pulmonares afectados } \\
\text { en tomografía de tórax inicial }\end{array}$ & Cuantitativa discreta & Escala de razón & 0-5 acorde a lóbulos afectados \\
\hline Enfisema pulmonar & Cualitativa nominal & Escala nominal & $\begin{array}{l}\text { 0: No } \\
\text { 1: Sí }\end{array}$ \\
\hline Fibrosis pulmonar & Cualitativa nominal & Escala nominal & $\begin{array}{l}\text { 0: No } \\
\text { 1: Sí }\end{array}$ \\
\hline Días de estancia intrahospitalaria & Cuantitativa discreta & Escala de razón & Numérico \\
\hline Ingreso a la UTI & Cualitativa nominal & Escala nominal & $\begin{array}{l}\text { 0: No } \\
\text { 1: Sí }\end{array}$ \\
\hline $\begin{array}{l}\text { Ventilación mecánica invasiva } \\
\text { (intubación) }\end{array}$ & Cualitativa nominal & Escala nominal & $\begin{array}{l}\text { 0: No } \\
\text { 1: Sí }\end{array}$ \\
\hline Días de intubación & Cuantitativa discreta & Escala de razón & Numérico \\
\hline Saturación de oxígeno $\left(\mathrm{SaO}_{2}\right)$ & Cuantitativa discreta & Escala de razón & Numérico \\
\hline $\begin{array}{l}\text { Relación } \mathrm{SaO}_{2} \text { y fracción } \\
\text { inspirada de oxígeno }\left(\mathrm{FiO}_{2}\right)\end{array}$ & Cuantitativa discreta & Escala de razón & Numérico \\
\hline Interleucina 6 (IL-6) & Cuantitativa discreta & Escala de razón & Numérico \\
\hline
\end{tabular}


Continúa la Tabla 1: Variables de estudio.

\begin{tabular}{|l|l|l|l|}
\hline Variable & Tipo de variable & Escala de medición & Unidad de medición \\
\hline Proteína C reactiva & Cuantitativa discreta & Escala de razón & Numérico \\
\hline Dímero D & Cuantitativa discreta & Escala de razón & Numérico \\
\hline Procalcitonina & Cuantitativa discreta & Escala de razón & Numérico \\
\hline Linfocitos & Cuantitativa discreta & Escala de razón & Numérico \\
\hline Ferritina & Cuantitativa discreta & Escala de razón & Numérico \\
\hline IDSA/ATS & Cualitativa nominal & Escala nominal & $\begin{array}{l}0: \text { No ingresa a la UTI } \\
1: \text { Ingresa a la UTI 1 criterio mayor } \\
2: \text { Ingresa a la UTI 3 criterios menores }\end{array}$ \\
\hline MuLBSTA & Cuantitativa discreta & Escala de razón & Numérico y porcentaje de mortalidad \\
\hline PSI & Cuantitativa discreta & Escala de razón & $\begin{array}{l}\text { Puntaje, clase de riesgo y porcentaje de } \\
\text { mortalidad }\end{array}$ \\
\hline $\begin{array}{l}\text { Volumen total de } \\
\text { parénquima pulmonar }\end{array}$ & Cuantitativa continua & Escala de razón & Porcentaje \\
\hline $\begin{array}{l}\text { Volumen de parénquima } \\
\text { pulmonar con vidrio esmerilado }\end{array}$ & Cuantitativa continua & Escala de razón & Porcentaje \\
\hline $\begin{array}{l}\text { Volumen de parénquima } \\
\text { pulmonar con consolidación }\end{array}$ & Cuantitativa continua & Escala de razón & Porcentaje \\
\hline $\begin{array}{l}\text { Volumen de parénquima } \\
\text { pulmonar bien ventilado }\end{array}$ & Cuantitativa continua & Escala de razón & Porcentaje \\
\hline $\begin{array}{l}\text { Índice de severidad por } \\
\text { volumetría (\%) }\end{array}$ & Cuantitativa discreta & Escala ordinal & $\begin{array}{l}0: 0 \\
1:(1-25) \\
2:(26-50) \\
3:(51-75) \\
4:(76-100)\end{array}$ \\
\hline
\end{tabular}

RT-PCR = reacción en cadena de la polimerasa transcriptasa reversa en tiempo real, UTI = Unidad de Terapia Intensiva, IL-6 = interleucina 6, IDSA/ATS = Sociedad Americana de Enfermedades Infecciosas/Sociedad Torácica Americana, PSI = índice de severidad de neumonía.

tórax, midiendo el volumen afectado por vidrio esmerilado y consolidaciones entre la totalidad del pulmón. El porcentaje se clasificó en cuatro grados de acuerdo con el nivel de afectación. Grado 1 corresponde a una afectación de 1 a 25\%, grado 2 una afectación de 26 a 50\%, grado 3 una afectación de 51 a $75 \%$ y, por último, grado 4 corresponde a una afectación de 76 a 100\%. Con base en estos grados se realizó una prueba de Kruskal-Wallis que determinó que la diferencia de las frecuencias de pacientes con ingreso a terapia intensiva, comorbilidades, clasificación IDSA/ATS, puntaje de PSI para la clase de gravedad, los lóbulos pulmonares afectados y el desenlace fueron estadísticamente significativos $(p<0.01)$.

Se realizó una prueba de correlación de Spearman en la que se determinó una correlación positiva entre el porcentaje total de parénquima pulmonar afectado con el índice de severidad por tomografía $(r=0.827)$, días de intubación $(r=0.437)$, puntaje de PSI $(r=0.4), \mathrm{FiO}_{2}(r=0.318)$, IL-6 $(r=0.534)$, PCR $(r=0.502)$, PCT $(r=0.509)$, ferritina $(r=0.507)$; así como una correlación negativa con $\mathrm{SaO}_{2} / \mathrm{FiO}_{2}(r=-0.393)$.

\section{Ingreso a Terapia Intensiva}

Se realizó una prueba de ANOVA para determinar si hay una diferencia significativa de porcentaje de parénquima afectado, puntaje de PSI y valores de laboratorio al comparar a los pacientes que ingresaron a terapia intensiva en comparación con los que tuvieron un internamiento fuera de esta área. Se determinó una diferencia significativa de las medias en cuanto al porcentaje de parénquima afectado $(p<0.001)$, puntaje PSI $(p<0.001)$, IL-6 ( $p<0.001)$, PCR $(p=0.03)$, dímero $D(p=0.01)$, PCT $(p<0.001)$, linfocitos $(p=0.03)$, ferritina $(p<0.01)$.

En cuanto a variables como edad, índice de severidad por tomografía, porcentaje de parénquima afectado, MuLBSTA y PSI, sólo el porcentaje total de parénquima afectado mostró un área bajo la curva aceptable de 0.739 con un punto de corte de $18 \%$ para tener una sensibilidad de 0.723 y una especificidad de 0.55. Mientras que las variables de edad, índice de severidad por tomografía, 
MuLBSTA y PSI tuvieron áreas bajo la curva pobres de $0.647,0.7,0.64$ y 0.7 , respectivamente.

\section{Variables durante la hospitalización (intubación y días de estancia intrahospitalaria)}

En cuanto a los pacientes que fueron intubados el número de días de estancia hospitalaria mostró correlación leve con el índice de severidad por tomografía $(r=0.409)$, volumen total de parénquima afectado $(r=473)$, puntaje

Tabla 2: Características demográficas.

\begin{tabular}{|c|c|}
\hline & $\mathrm{n}(\%)$ \\
\hline $\begin{array}{l}\text { Sexo } \\
\text { Hombre } \\
\text { Mujer }\end{array}$ & $\begin{array}{r}155(66.5) \\
78(33.5)\end{array}$ \\
\hline $\begin{array}{l}\text { Origen } \\
\text { Triaje } \\
\text { Ambulatorio } \\
\text { Prequirúrgico }\end{array}$ & $\begin{array}{c}218(93.6) \\
11(4.7) \\
4(1.7)\end{array}$ \\
\hline $\begin{array}{l}\text { Hallazgos por tomografía } \\
\text { Vidrio esmerilado } \\
\text { Consolidación } \\
\text { Crazy paving } \\
\text { Indeterminado } \\
\text { Atípico } \\
\text { Negativo }\end{array}$ & $\begin{aligned} 141 & (60.5) \\
37 & (15.9) \\
34 & (14.6) \\
3 & (1.3) \\
8 & (3.4) \\
10 & (4.3)\end{aligned}$ \\
\hline $\begin{array}{l}\text { Lóbulos afectados } \\
\qquad 0 \\
1 \\
2 \\
3 \\
4 \\
5\end{array}$ & $\begin{aligned} 10 & (4.3) \\
11 & (4.7) \\
4 & (1.7) \\
8 & (3.4) \\
18 & (7.7) \\
182 & (78.1)\end{aligned}$ \\
\hline Enfisema pulmonar & $24(10.3)$ \\
\hline Fibrosis pulmonar & $14(6.0)$ \\
\hline Derrame pleural & $28(12.0)$ \\
\hline Manejo ambulatorio & $33(14.2)$ \\
\hline UTI & $93(39.9)$ \\
\hline $\begin{array}{l}\text { IDSA/ATS ingreso a la UTI } \\
\text { No ingresa } \\
\text { Un criterio mayor } \\
\text { Tres criterios menores }\end{array}$ & $\begin{array}{r}154(66.1) \\
53(22.3) \\
27(11.6)\end{array}$ \\
\hline $\begin{array}{l}\text { Porcentaje de parénquima } \\
\text { afectado en grados (\%) } \\
\text { Grado } 1(0-25) \\
\text { Grado } 2(26-50) \\
\text { Grado } 3(51-75) \\
\text { Grado } 4(76-100)\end{array}$ & $\begin{array}{r}128(54.9) \\
52(22.3) \\
25(10.7) \\
3(1.3)\end{array}$ \\
\hline
\end{tabular}

UTI = Unidad de Terapia Intensiva, IDSA/ATS = Sociedad Americana de Enfermedades Infecciosas/Sociedad Torácica Americana.
PSI $(r=0.437)$, IL-6 $(r=0.436)$, ferritina $(r=0.333)$ y PCT $(r=0.331)$. Se realizó una regresión multivariada con dichas variables para estimar los días de intubación. Se obtuvo un modelo no significativo con una $\mathrm{R}$ de 0.504 , R cuadrado de 0.254 y $R$ cuadrado ajustado de 0.232 .

Respecto al tiempo de internamiento de los pacientes, se observó una correlación leve con el porcentaje total de parénquima respetado $(r=0.375)$, ferritina $(r=0.349)$, PCT $(r=0.343)$ e IL-6 $(r=0.448)$. Se realizó una regresión multivariada con dichas variables para estimar los días de internamiento. Se obtuvo un modelo no significativo con una $R$ de 0.398 , R cuadrado de 0.158 y $R$ cuadrado ajustado de 0.139 .

\section{Desenlace}

En cuanto al desenlace de los pacientes se realizó una prueba de ANOVA tomando en cuenta el porcentaje de parénquima afectado, índice de severidad por tomografía, puntajes PSI y MuLBSTA, así como las variables de laboratorio. Se obtuvieron valores estadísticamente significativos para el porcentaje total de parénquima respetado $(p=0.007)$, PSI $(p<0.001)$, días de intubación $(p<0.001)$, días de estancia hospitalaria $(p=0.04)$, IL-6 $(p=0.04)$, dímero $\mathrm{D}(\mathrm{p}<0.001)$ y PCT.

\section{DISCUSIÓN}

\section{Grado de afectación del parénquima pulmonar}

La estratificación por grados de afección pulmonar en pacientes con neumonía por COVID-19 resultó de una búsqueda por un predictor imagenológico para desenlaces adversos, como la muerte; y fue descrito inicialmente por Colombi et al. quienes utilizaron un sistema semicuantitativo por computadora para valorar las áreas de pulmón que estaban respetadas y las áreas afectadas por vidrio despulido y consolidación. Al seguir este y otros trabajos que han sido publicados durante la pandemia se realizó la estratificación de la afección del parénquima pulmonar por grados, como ya hemos mencionado, y con base en éstos se encontró una diferencia estadísticamente significativa para las frecuencias de los lóbulos pulmonares afectados. Este es un hallazgo que resulta lógico, ya que a mayor afección del parénquima pulmonar se encontrará afección de mayor cantidad de lóbulos. También existió una diferencia estadísticamente significativa para la escala clínica con puntaje de PSI (que sobre todo es útil para valorar mortalidad) para pacientes ingresados a la UTI y para los distintos desenlaces. Estos puntos serán comentados en los apartados subsecuentes.

Uno de los hallazgos más relevantes de este estudio fue la correlación positiva entre el porcentaje total del parénquima pulmonar afectado y el índice de severidad 
Tabla 3: Valores de laboratorio.

\begin{tabular}{|c|c|}
\hline & Muestra total, mediana (RIC) \\
\hline $\mathrm{SAO}_{2}$ & $94(90-96)$ \\
\hline $\mathrm{FiO}_{2}$ & $0.32(0.21-0.5)$ \\
\hline $\mathrm{SAO}_{2}$ & $306(191-433)$ \\
\hline $\mathrm{IL}-6$ & $63.5(28.9-170.2)$ \\
\hline $\mathrm{PCR}$ & $9.47(3-18.8)$ \\
\hline Dímero D & $909(570-1,407)$ \\
\hline PCT & $0.15(0.08-0.44)$ \\
\hline Linfocitos 10^3/uL & $1(0.76-1.47)$ \\
\hline Ferritina & $983(501-1,820)$ \\
\hline
\end{tabular}

$\mathrm{RIC}=$ rango intercuartílico

por tomografía. Como mencionamos previamente, existen esfuerzos por encontrar una escala de severidad por tomografía que sea útil para emplear en pacientes con neumonía por COVID-19, y dentro de estos esfuerzos se ha optado por emplear escalas previamente aceptadas y estudiadas para otras neumonías. Sin embargo, no existe un estudio que muestre una correlación clara entre los hallazgos del parénquima afectado y algún índice de severidad. El CT-SS empleado en este estudio sí mostró una correlación positiva con los diferentes grados de afección pulmonar.

Además, se encontró una correlación positiva entre el grado de afección pulmonar y los reactantes de fase aguda que en la actualidad han mostrado utilidad para el manejo de los pacientes con neumonía por COVID-19, como son la IL-6, PCR, procalcitonina y ferritina. Estos hallazgos también se discutirán a continuación.

\section{Ingreso a Terapia Intensiva}

El análisis cuantitativo por tomografía al parénquima afectado en pacientes COVID-19 ha sido asociado al riesgo de complicaciones y atención en unidades de terapia intensiva. En el caso del estudio de Lanza et al. ${ }^{20}$ utilizaron el mismo software que nosotros para el cálculo total del parénquima afectado. En su caso, determinaron que pacientes con afectación entre seis a $23 \%$ tienen riesgo de necesitar terapia de oxigenación, y por arriba de $23 \%$ están en riesgo de intubación. En nuestro caso, realizamos una prueba ANOVA para la comparación de las medias en pacientes internados fuera y dentro de la UTI, se hizo una transformación previa a la distribución normal para la comparación de las medias que fueron de 20 y 36\% para pacientes internados en hospitalización normal y aquellos internados dentro de terapia intensiva, respectivamente. Esto es concordante con lo reportado por Lanza et al. ya que estos dos grupos requirieron terapia de oxigenación y tratamiento intrahospitalario.
En estudios previos se ha investigado la asociación de distintos parámetros de laboratorio en pacientes con neumonía por COVID-19 y la severidad de la enfermedad. ${ }^{21,22}$ En cuanto a los niveles de ferritina se ha visto que los pacientes con enfermedad severa por COVID-19 tienen un aumento en comparación con los pacientes con enfermedad leve o moderada, esto debido a ser un mediador clave en la regulación del sistema inmune. En nuestro caso, los niveles de ferritina tuvieron un desempeño moderado en discernir si un paciente debe ser tratado o no en la UTI; en el análisis de la curva ROC tuvieron un área bajo de curva de 0.699. La media de niveles de ferritina de los pacientes dentro y fuera de terapia intensiva fue de 2,391 y 1,042, respectivamente. Para predecir el ingreso a la UTI, el índice de severidad por tomografía no arrojó resultados significativos, ya que mostró un área bajo la curva de 0.7. Hay estudios que difieren de este hallazgo, como reportan Yang et al. ${ }^{12}$ quienes abogan que esta sería una variable por considerarse para pacientes en riesgo de intubación y por ende que requieren de ingreso a la UTI. Sin embargo, el porcentaje total de parénquima afectado sí mostró una correlación con un área bajo la curva de 0.74 , con una sensibilidad de 0.72 y especificidad de 0.5 para un punto de corte de $18 \%$. Li et al. concuerdan con nuestros hallazgos y proponen que el uso de una herramienta para cuantificar el porcentaje de parénquima pulmonar afectado es útil para predecir a pacientes que requieren de intubación y, por lo tanto, de ingreso a la UTI.

Variables de hospitalización: intubación y días de estancia intrahospitalaria

A la fecha no existe un estudio que reporte una correlación de los días de intubación con el volumen total del parén-

Tabla 4: Valores volumétricos.

\begin{tabular}{|l|c|}
\hline & $\begin{array}{c}\text { Muestra total, } \\
\text { mediana (RIC) }\end{array}$ \\
\hline${ }^{*}$ Volumen pulmonar total & $3,043(2,271-3,877)$ \\
\hline${ }^{*}$ Volumen parénquima respetado & $2,414(1,428-3,265)$ \\
\hline${ }^{*}$ Volumen vidrio esmerilado & $422(181-747)$ \\
\hline${ }^{*}$ Volumen consolidación & $133(46-340)$ \\
\hline${ }^{*}$ Volumen total de parénquima afectado & $601(230-1,004)$ \\
\hline Porcentaje total de parénquima afectado & $19.2(8.8-39.4)$ \\
\hline${ }^{\ddagger}$ Atenuación parénquima respetado & $-833(-856--798)$ \\
\hline${ }^{\ddagger}$ Atenuación de consolidación & $-74(-578--470)$ \\
\hline $\begin{array}{l}{ }^{\ddagger} \text { Atenuación parénquima con vidrio } \\
\text { esmerilado, (media } \pm \text { DE) }\end{array}$ & $-525 \pm 87.2$ \\
\hline
\end{tabular}

$\mathrm{RIC}=$ rango intercuartílico, ${ }^{\star}=$ unidad medida en centímetros cúbicos, ${ }^{\ddagger}=$ valores medidos en unidades Hounsfield, DE = desviación estándar. 
quima pulmonar afectado. Sin embargo, hay reportes y propuestas en las que se identifica el porcentaje de afección pulmonar como un valor predictivo positivo de mortalidad y de probabilidad de que un paciente requiera intubación.

En nuestro estudio se observó una correlación positiva leve entre los pacientes con intubación y el índice de severidad por tomografía $(r=0.409)$, así como el volumen total del parénquima afectado $(r=0.473)$. Como habíamos mencionado previamente, Lanza et al. realizaron un estudio retrospectivo unicéntrico en 222 pacientes hospitalizados con diagnóstico de COVID-19 en el que destaca que $75 \%$ recibió soporte de oxigenación. Como nosotros, este estudio empleó un análisis cuantitativo de tomografía de tórax con un método semiautomático, en el que se realizó segmentación pulmonar de acuerdo con intervalos de unidades Hounsfield (UH) y se estimó el volumen pulmonar comprometido como el predictor de resultado más preciso. Los valores del volumen pulmonar comprometido en el rango de 6-23\% incrementó el riesgo de soporte ventilatorio, los valores superiores a $23 \%$ presentaron riesgo de intubación y se consideró como un factor de riesgo de mortalidad intrahospitalaria $(p<0.001)$. En nuestro estudio vemos que los hallazgos correlacionan con lo encontrado por Lanza et al.

Existieron otras variables que también mostraron una correlación con los días de intubación como fue el puntaje PSI que ha mostrado una alta predicción de mortalidad. Contrario a este resultado, en el reporte por Clemente et al. ${ }^{23}$ se ha visto que este método no es bueno para discernir si un paciente debe ser manejado en terapia intensiva o requerirá el uso de ventilación mecánica.

Para los días de estancia intrahospitalaria se observó una correlación leve con el porcentaje total del parénquima respetado, mas no con el afectado como lo encontrado para el ingreso a la UTI y el uso de ventilación mecánica. No hay reportes que mencionen esta correlación encontrada con el volumen respetado de parénquima pulmonar. No existió correlación con el índice de severidad por tomografía. También se tomaron en cuenta los niveles de reactantes de fase aguda, como lo mencionamos en el apartado anterior, tales como la IL-6, procalcitonina y ferritina, con las cuales observamos una correlación leve entre estos marcadores y los días de internamiento.

\section{Correlación con el desenlace}

Francone et al. reportan una correlación con la extensión de la afección del parénquima estimado por tomografía y la mortalidad a corto plazo durante un período de observación de 24 días, siendo el valor de corte de 18\% como altamente predictivo a corto plazo. ${ }^{24} \mathrm{Al}$ igual que este estudio, en el nuestro encontramos que existe una diferencia significativa de las medias del parénquima pulmonar respetado entre los pacientes egresados y los pacientes con desenlace fatal. El índice de severidad no mostró una diferencia significativa entre estos dos grupos. Llama la atención que no se encontraron diferencias significativas entre los grupos del desenlace para las escalas clínicas de mortalidad (PSI y MuLBSTA), que en la actualidad son empleadas para valorar a estos pacientes y que su función principal es predecir mortalidad a corto plazo.

Asimismo, se encontró una diferencia significativa de las medias al comparar a los reactantes de fase aguda con el grupo de pacientes que egresó y los que fallecieron, lo cual coincide con lo reportado en la literatura actual. ${ }^{25}$ Múltiples estudios han demostrado que se pueden utilizar estas pruebas de laboratorio como marcadores pronósticos de riesgo y mortalidad en pacientes con COVID-19, así como el espectro de biomarcadores actualmente disponibles es suficiente para completar este propósito. ${ }^{26-29}$ Skevaki et al. realizaron una revisión sistemática de la literatura en la que encontraron una correlación directa entre la concentración de ferritina sérica y la mortalidad, así como que concentraciones de procalcitonina (por lo general $\geq 0.05 \mathrm{ng} / \mathrm{mL}$ ) pueden distinguir significativamente entre los casos de pacientes no graves, graves y aquellos con desenlace fatal, por lo que funcionan como buenos marcadores pronósticos de la enfermedad. ${ }^{30}$

\section{CONCLUSIÓN}

Existe una correlación positiva entre el porcentaje de afección pulmonar medida de forma semicuantitativa y el puntaje de severidad evaluado en este estudio (CT-SS).

Para el pronóstico de ingreso a la UTI, el CT-SS no es útil. Aunque, el porcentaje de afección pulmonar mostró una sensibilidad de $72 \%$ y una especificidad de $55 \%$ para predecir el ingreso a la UTI. Otra variable útil para discernir pacientes que requieren UTI fue la ferritina.

Con respecto a la intubación, el CT-SS y el porcentaje de parénquima afectado mostraron una correlación leve, por lo que ambas pueden ser utilizadas para predecir pacientes que requieren ventilación mecánica invasiva. La clase de PSI también fue otra variable que se encontró en correlación con la intubación, aunque no es una escala diseñada para este fin.

Para predecir los días de estancia intrahospitalaria se encontró una correlación leve con el grado de volumen pulmonar respetado y podría emplearse en la práctica clínica. El CT-SS no mostró resultados estadísticamente significativos.

En cuanto a mortalidad, existe una diferencia significativa entre las medias encontradas en el grupo de pacientes egresados y aquellos con desenlace fatal para los reactantes de fase aguda y el porcentaje de volumen pulmonar respetado. El CT-SS no mostró resultados estadísticamente significativos. 
Con lo encontrado en este estudio concluimos que el CT-SS podría ser empleado para predecir pacientes que requerirán ventilación mecánica, pero no fue útil para el resto de las variables estudiadas. No obstante, el porcentaje de volumen pulmonar afectado y respetado fue útil para predecir el ingreso a la UTI, el uso de ventilación mecánica, los días de estancia intrahospitalaria y el desenlace (pacientes egresados o con desenlace fatal).

\section{Agradecimientos}

Queremos reconocer y agradecer la labor realizada por el personal de salud en México durante la pandemia por COVID-19.

\section{REFERENCIAS}

1. Zhu N, Zhang D, Wang W, Li X, Yang B, Song J, et al. A novel coronavirus from patients with pneumonia in China, 2019. N Engl J Med. 2020;382(8):727-733. https://doi.org/10.1056/nejmoa2001017.

2. Johns Hopkins: COVID-19 Dashboard by the Center for Systems Science and Engineering (CSSE) at Johns Hopkins University (JHU). https://coronavirus.jhu.edu/map.html.

3. Wu Z, McGoogan JM. Characteristics of and important lessons from the coronavirus disease 2019 (COVID-19) outbreak in China: summary of a report of 72314 cases from the Chinese Center for Disease Control and Prevention. JAMA. 2020;323(13):1239-1242. https://doi. org/10.1001/jama.2020.2648.

4. Li Q, Guan X, Wu P, Wang X, Zhou L, Tong Y, et al. Early transmission dynamics in Wuhan, China, of novel coronavirus-infected pneumonia. N Engl J Med. 2020;382(13):1199-1207. https://doi.org/10.1056/ nejmoa2001316.

5. Huang P, Liu T, Huang L, Liu H, Lei M, Xu W, et al. Use of chest CT in combination with negative RT-PCR assay for the 2019 novel coronavirus but high clinical suspicion. Radiology. 2020;295(1):22-23. https://doi.org/10.1148/radiol.2020200330.

6. Fang $Y$, Zhang H, Xie J, Lin M, Ying L, Pang P, et al. Sensitivity of chest CT for COVID-19: comparison to RT-PCR. Radiology. 2020;296(2):E115-E117. https://doi.org/10.1148/radiol.2020200432.

7. Pan Y, Guan H, Zhou S, Wang Y, Li Q, Zhu T, et al. Initial CT findings and temporal changes in patients with the novel coronavirus pneumonia (2019-nCoV): a study of 63 patients in Wuhan, China. Eur Radiol. 2020;30(6):3306-3309. https://doi.org/10.1007/s00330-02006731-x.

8. He F, Deng Y, Li W. Coronavirus disease 2019: What we know? J Med Virol. 2020;92(7):719-725. https://doi.org/10.1002/jmv.25766.

9. Rubin GD, Ryerson CJ, Haramati LB, Sverzellati N, Kanne JP, Raoof $\mathrm{S}$, et al. The role of chest imaging in patient management during the COVID-19 pandemic: A Multinational Consensus Statement from the Fleischner Society. Radiology. 2020;296(1):172-180. https://doi. org/10.1148/radiol.2020201365.

10. Simpson S, Kay FU, Abbara S, Bhalla S, Chung JH, Chung M, et al. Radiological Society of North America Expert Consensus Statement on Reporting Chest CT Findings Related to COVID-19. Endorsed by the Society of Thoracic Radiology, the American College of Radiology, and RSNA-Secondary publication. J Thorac Imaging. 2020;35(4):219227. https://doi.org/10.1097/rti.0000000000000524.
11. Koo JH, Lim S, Choe J, Choi S-H, Sung H, Do K-H. Radiographic and CT features of viral pneumonia. Radiographics. 2018;38(3):719-739. https://doi.org/10.1148/rg.2018170048.

12. Yang R, Li X, Liu H, Zhen Y, Zhang X, Xiong Q, et al. Chest CT severity score: an imaging tool for assessing severe COVID-19. Radiology: Cardiothoracic Imaging. 2020;2(2):e200047. https://doi.org/10.1148/ ryct.2020200047

13. Li K, Fang Y, Li W, Pan C, Qin P, Zhong Y, et al. CT image visual quantitative evaluation and clinical classification of coronavirus disease (COVID-19). Eur Radiol. 2020;30(8):4407-4416. https://doi. org/10.1007/s00330-020-06817-6.

14. Colombi D, Bodini FC, Petrini M, Maffi G, Morelli N, Milanese G, et al. Well-aerated lung on admitting chest $C T$ to predict adverse outcome in COVID-19 pneumonia. Radiology. 2020;296(2):E86-E96. https:// doi.org/10.1148/radiol.2020201433.

15. Ichikado K, Muranaka H, Gushima Y, Kotani T, Nader HM, Fujimoto $\mathrm{K}$, et al. Fibroproliferative changes on high-resolution CT in the acute respiratory distress syndrome predict mortality and ventilator dependency: a prospective observational cohort study. BMJ Open. 2012;2(2):e000545. https://doi.org/10.1136/ bmjopen-2011-000545.

16. Mandell LA, Wunderink RG, Anzueto A, Bartlett JG, Campbell GD, Dean NC, et al. Infectious Diseases Society of America/American Thoracic Society consensus guidelines on the management of community-acquired pneumonia in adults. Clin Infect Dis. 2007;44(Suppl 2):S27-S72. https://doi.org/10.1086/511159.

17. Guo L, Wei D, Zhang X, Wu Y, Li Q, Zou M, et al. Clinical features predicting mortality risk in patients with viral pneumonia: The MuLBSTA score. Front Microbiol. 2019;10:2752. https://doi. org/10.3389/fmicb.2019.02752.

18. Lim WS, van der Eerden MM, Laing R, Boersma WG, Karalus N, Town Gl, et al. Defining community acquired pneumonia severity on presentation to hospital: an international derivation and validation study. Thorax. 2003;58(5):377-382. https://doi.org/10.1136/ thorax.58.5.377.

19. Du Y, Tu L, Zhu P, Mu M, Wang R, Yang P, et al. Clinical features of 85 fatal cases of COVID-19 from Wuhan. A retrospective observational study. Am J Respir Crit Care Med. 2020;201(11):1372-1379. https:// doi.org/10.1164/rccm.202003-05430c.

20. Lanza E, Muglia R, Bolengo I, Santonocito OG, Lisi C, Angelotti G, et al. Quantitative chest CT analysis in COVID-19 to predict the need for oxygenation support and intubation. Eur Radiol. 2020;30(12):67706778. https://doi.org/10.1007/s00330-020-07013-2.

21. Coomes EA, Haghbayan H. Interleukin-6 in Covid-19: a systematic review and meta-analysis. Rev Med Virol. 2020;30(6):1-9. https://doi. org/10.1002/rmv.2141.

22. Herold T, Jurinovic V, Arnreich C, Lipworth BJ, Hellmuth JC, von Bergwelt-Baildon $M$, et al. Elevated levels of IL- 6 and CRP predict the need for mechanical ventilation in COVID-19. J Allergy Clin Immunol. 2020;146(1):128-136.e4. https://doi.org/10.1016/j. jaci.2020.05.008.

23. Clemente MMG, Herrero-Huertas J, Fernández-Fernandez A, Muñoz CDLE, Rodríguez AIE, Martínez LP, et al. Assessment of risk scores in COVID-19. Int J Clin Pract. 2020;e13705. https://doi.org/10.1111/ ijcp.13705.

24. Francone M, lafrate F, Masci GM, Coco S, Cilia F, Manganaro L, et al. Chest CT score in COVID-19 patients: correlation with disease severity and short-term prognosis. Eur Radiol. 2020;30(12):6808-6817. https://doi.org/10.1007/s00330-020-07033-y. 
Neumol Cir Torax. 2021; 80 (1): 19-28

25. Vargas-Vargas M, Cortés-Rojo C. Ferritin levels and COVID-19. Rev Panam Salud Publica. 2020;44:e72. https://doi.org/10.26633/ rpsp.2020.72.

26. Skevaki C, Fragkou PC, Cheng C, Xie M, Renz H. Laboratory characteristics of patients infected with the novel SARS-CoV-2 virus. J Infect. 2020;81(2):205-212. https://doi.org/10.1016/j.jinf.2020.06.039.

27. Chen $T$, Wu D, Chen H, Yan W, Yang D, Chen G, et al. Clinical characteristics of 113 deceased patients with coronavirus disease 2019: retrospective study. BMJ. 2020;368:m1091. https://doi. org/10.1136/bmj.m1091.

28. Zhou F, Yu T, Du R, Fan G, Liu Y, Zhibo L, et al. Clinical course and risk factors for mortality of adult inpatients with COVID-19 in Wuhan,
China: a retrospective cohort study. Lancet. 2020;395(10229):10541062. https://doi.org/10.1016/s0140-6736(20)30566-3.

29. Chen G, Wu Di, Guo W, Cao Y, Huang D, Wang H, et al. Clinical and immunological features of severe and moderate coronavirus disease 2019. J Clin Invest. 2020;130(5):2620-2629. https://doi.org/10.1172/jci137244.

30. Tan L, Wang Q, Zhang D, Ding J, Huang Q, Tang Y-Q, et al. Lymphopenia predicts disease severity of COVID-19: a descriptive and predictive study. Signal Transduct Target Ther. 2020;5(1):33. https://doi.org/10.1038/s41392-020-0148-4.

Conflicto de intereses: Los autores declaran no tener conflicto de intereses. 